\title{
Early Detection of Exercise - Associated Muscle cramp
}

\author{
Sinthia $P^{1}$, M. Malathi ${ }^{2}$, S. Nagarajan ${ }^{3 a}$, Anitha Juiette ${ }^{3 b}$, Nalini.M ${ }^{4}$ \\ 1 Associate Professor, Department of Biomedical Engineering, Saveetha Engineering \\ College \\ 2 Associate Professor, Department of Electronics and Communication Engineering, \\ Rajalakshmi Institute of Technology. \\ 3 a Associate Professor, Department of Electronics and Communication Engineering, \\ Saveetha Engineering College. \\ 3 b Associate Professor, Department of Electronics and Communication Engineering, Loyola- \\ ICAM College of Engineering and Technology. \\ 4 Assistant Professor, Department of Computer Science and Engineering, Saveetha School of \\ Engineering.
}

\begin{abstract}
One of the deep and painful involuntary contractions of skeletal muscle is muscle cramp which takes place during various other conditions. The origin for the cramps that occur during or soon after exercise and the appropriate remedies continue to prove uncertain. Soon after an implant process and forceful workout, past voluntary dehydration cramps occur at many sections. The process of identifying the type of cramp is time consuming and treatment process to cramps is also quite tedious. If not treated at the right time, Muscle cramps may cause vigorous side effects and worsening in day to day physiological activities. The main motive of this vest is to locate and identify the cramp and alert so that further injury can be detected. It would also be helpful in preventing the person from further muscular cramps and other complications. EMG signals which are obtained from adhesive electrodes are amplified by using IC741 (op-amp) with instrumentation amplifier configuration.LM35 temperature sensor is used to monitor the temperature at specified locations. Wherever the cramp occurring possibilities are high. Flex sensor is used to identify the abnormal contraction and relaxation muscles in upper limbs. The three input bio potential signals are fed to the micro controller (Arduino UNO). The main objective of this system is to provide a comfortable vest which would monitor the cramps occurring in athletes whenever it occurs. Therefore with the help of this wearable device muscle cramps occurring at upper limbs can be detected and further injuries, complications such as fractures can be reduced.
\end{abstract}

Key Words: Muscle cramps, spasms, Flex sensor, Vest

\section{INTRODUCTION}

An unexpected and spontaneous retrenchment of one or more of your muscles is generally termed as muscle cramps or muscle spasms. Sometimes when a person is awakened in the middle of his deep sleep during the night or affected by a sudden charley horse, he may be subjected to muscle cramps that causes severe pain. Consequently this causes uncomfortable muscle contractions leading to immobility. Prolonged work out or bodily work especially in 
summer can also cause muscle cramps. Muscle cramps can also be a result of usage of some drugs and certain health conditions. Muscle cramps can be treated at home with health-care procedures if diagnosed at the right time. The most common location of muscle convulsions is in the gastrocnemius muscles, to be specific the cramps are usually located in the calf. In addition to a strong pain, the patient might also experience or observe a rigid swelling of muscle tissue underneath the affected skin. These cramps or spasms usually disappear on their own. The other causes of cramps are overdoing of work-outs, dehydration, muscle strain or staying in the same spot for an extended phase. In a lot of instances, however, the reason isn't identified. Although most muscle cramps does not seem to cause harm, sometimes the causes for cramps may be associated to a medical condition of the person which exists already.

- Ageing. Aged people drop muscle potent, as a result of this condition, the left over muscle can get stressed excessively further more.

- Dehydration. Fatigue and dehydration occurring in athletes while taking part in hot-weather sports commonly increase the risk of developing muscle cramps.

- Pregnancy. Muscle cramps are frequent indications of gestation.

- Health ailments. Some medical illness like diabetes, or nerve, liver or thyroid disorders can cause muscle cramps.

\section{LITERATURE REVIEW}

1. MUSCLE CRAMPS TIMOTHY M. MILLER, MD, PhD, 1 and ROBERT B. LAYZER, MD2 1 Department of Neuroscience, University of California, San Diego, 9500 Gilman Drive, La Jolla, California 92093-0670, USA 2 Department of Neurology, University of California, San Francisco, California, USA Accepted 24 February 2005. Muscle cramps are a widespread difficulty whose characteristics are a unexpected, excruciating, uncontrolled retrenchment of muscle. These existent muscle spasm, which are initiated from circumferential nerves, can sometimes be differentiated from other muscular discomfort or spasm. Parameters used to determine the sources for these conditions are past medical history, bodily investigation, and a constricted laboratory tests. Despite the fact that these cramps are "benign" in nature, a lot of patients come across the symptoms which remain very uncomfortable.

\section{Origin and development of muscle cramps MA Minetto, $\underline{\text { A Holobar, }} \underline{\text { A Botter- }}$ Exercise and sports 2013}

This paper proposes a technique of door unlocking scheme for the system containing numerous doors. This type of system can be used to access in a certain area. E.g. laboratory or library. The proposed system mainly based on arduino microcontroller. It includes several components like RFID reader; passive RFID tags of Arduino IDE and process expansion setting are required for control. All these processes are possible by automatic synchronizations with a secured internet. 


\section{Exercise-Associated Muscle Cramps Causes, Treatment, and Prevention}

Kevin C. Miller, PhD, ATC, CSCS, ${ }^{* \dagger}$ Marcus S. Stone, PhD, ATC, ${ }^{\dagger}$ Kellie C. Huxel, PhD, ATC,${ }^{\S}$ and Jeffrey E. Edwards, PhD. Recreational and competitive athletes usually come across a frequent difficulty which is called as Exercise-associated muscle cramps (EAMC). In addition to the fact that these muscle cramps are very common and frequently occurring in athletes, their origin remains unidentified. Speculations for the basis of EAMC are principally based on informal and empirical examinations rather than stable investigatory proof. Therefore the remedial procedures and prevention methodologies for EAMC are very much ineffective because the clear reason always remains unidentified.

4.Exercise-induced muscle pain, soreness, and cramps.MP Miles, PM Clarkson - The Journal of sports medicine and 1994

There are totally three sorts of pain associated with work out and fitness. They are

1) Discomfort encountered at some stage in or immediately subsequent to the exercise phase.

2) Postponed onset muscle pain, and

3) Ache persuaded by muscle cramps.

Each pain is distinguished by a different time course and different etiology.

\section{METHODOLOGY}

\section{BLOCK DIAGRAM}

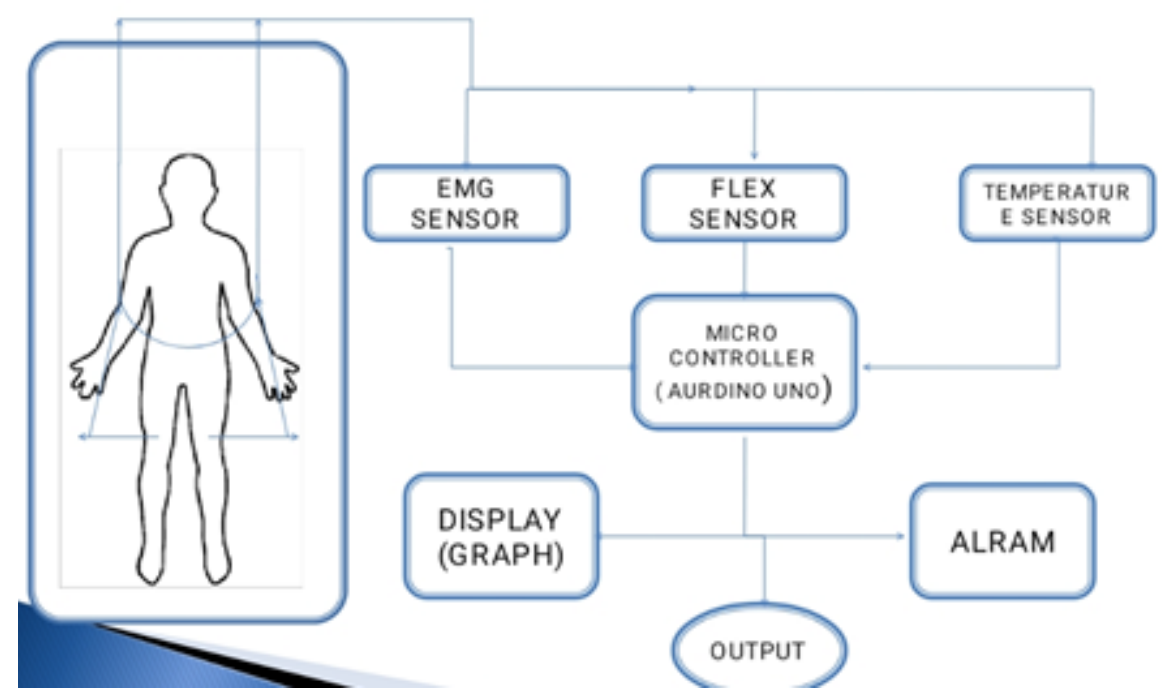

Fig.1 Block Diagram of the proposed system

The main motive of this vest is to locate and identify the cramp and alert so that further injury can be detected. It would also stop further muscular cramps and other complications.

EMG signals which are obtained from adhesive electrodes, are amplified by using IC741 (op-amp) with instrumentation amplifier configuration.LM35 temperature sensor is used to monitor the temperature at specified location where cramp occurring possibilities are high. Flex sensor is used to identify the abnormal contraction and relaxation muscles in upper limbs. This three input bio potential signals are fed to the micro controller (Arduino UNO). 
Calibration and alert signal will be activated and processed by the micro controller with the help of proper coding. These electronic components are insulated and fixed in a vest (unisex) where the damage of components and uncomfortable conditions occurring possibilities are very much reduced.

\section{i) FLEX SENSOR}

The device which is used to measure the degree of bending and deflection is called as Flex sensor. The commonly available two sizes of Flex sensor are 2.2 inch and the another is 4.5 inch. In spite of the dissimilarities in sizes, the fundamental role continues to be unchanged. Flex sensor can also be categorized depending upon the resistance such as low-level resistance, moderate resistance and high-level resistance types. The appropriate types of sensor can be chosen depending upon the requirement. FS-L-0055 is the flex sensor used and the size of the sensor is about 2.2. inch.

The resistance of the sensor terminal shows significant changes when it is twisted. The operation of Flex sensor is similar to that of a variable resistor whose terminal resistance gets high on bending the sensor. So the terminal resistance of the sensor rises depending upon the surface linearity. This fact is utilized in sensing the changes in linearity.

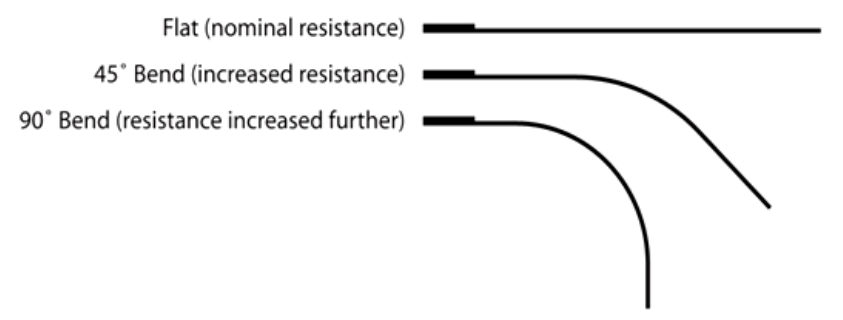

Fig.2 Variable resistance

Figure 2 shows that, when the flex sensor is having totally linear surface, the resistance will be nominal. When it is inclined at angle of $45^{\circ}$, the terminal resistance of the flex sensor gets enlarged to double times that of the before. And when the sensor is bent at $90^{\circ}$, the resistance gets increased to four times higher than that of the nominal resistance. So the resistance across the terminals gets high in a linear manner with bent angle. So in a way, we can say that the flex sensor converts flex angle to the corresponding resistance value.

For convenience, this resistance can be converted to the appropriate voltage value. For this purpose, the voltage divider circuit can be used.

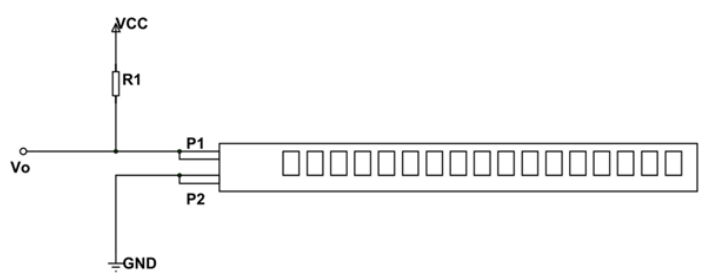

Fig.3. Circuit Diagram of Flex Sensor

This figure illustrates that, the resistance R1 here is invariant and flex sensor behaves like a variable resistance. The output signal is $\mathrm{V}_{\mathrm{o}}$ and thus it is the voltage across the flex sensor. 
Here,

$\mathrm{V}_{\mathrm{o}}=\mathrm{V}_{\mathrm{CC}}\left(\mathrm{R}_{\mathrm{x}} /\left(\mathrm{R}_{1}+\mathrm{R}_{\mathrm{x}}\right)\right)$.

$\mathrm{R}_{\mathrm{x}}$ - Resistance of the flex sensor

Now, there is an increase in the terminal resistance when the flex sensor is subjected to bending process..

The voltage divider circuit also exhibits this increase. With this increase, the reduction across the flex sensor gets expanded and consequently $V_{o}$ also increases. So as the bending of flex sensor gets high, the voltage $\mathrm{V}_{\mathrm{o}}$ increases linearly. This represents the voltage parameter corresponding to the flex.

This voltage parameter is taken and fed as input to ADC to acquire the digital value to be utilized for further processing.

\section{ii) TEMPERATURE SENSOR- LM35}

- LM35 is a device which is used to estimate temperature. It has an analog output signal corresponding to the measured temperature.

- This device produces output potential in Centigrade (Celsius) unit. No external calibration circuitry is required.

- LM35 exhibits a sensitivity of $10 \mathrm{mV} /$ degree Celsius. The increase in temperature produces corresponding increase in the output voltage. E.g. $250 \mathrm{mV}$ means $25^{\circ} \mathrm{C}$.

- LM35 acts as sensor consisting of a 3-terminal which is used to compute nearby temperature whose range is from $55{ }^{\circ} \mathrm{C}$ to $150{ }^{\circ} \mathrm{C}$. The temperature output potential produced by this sensor is much more accurate as compared to the output produced by the thermistor.

- LM35 is said to exhibit the properties of a precession Integrated circuit Temperature sensor, whose output voltage changes, depending upon the temperature surrounding the device. It seems to be very small and is also a cost efficient IC which can be utilized to determine temperature anywhere between $-55^{\circ} \mathrm{C}$ to $150^{\circ} \mathrm{C}$. Micro-controller consisting of an $\mathrm{ADC}$ function or any development platform like Arduino can be used as an interfacing platform.

- A regulated voltage like $+5 \mathrm{~V}\left(\mathrm{~V}_{\mathrm{S}}\right)$ can be applied to power the IC to the input terminal and the ground pin is connected to the zero potential layer of the circuit. Now, the temperature can be calculated in terms of voltage as shown in the below figure.

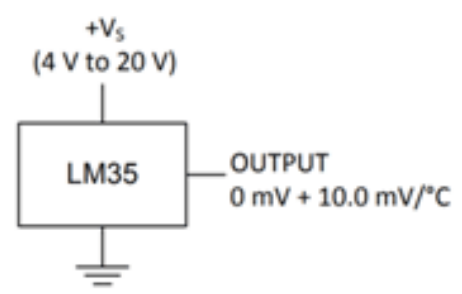

Fig.4. Temperature Sensor

At $\mathrm{i}^{\circ} \mathrm{C}$ the output voltage will also be $0 \mathrm{~V}$. The sensor shows an increase of $0.01 \mathrm{~V}(10 \mathrm{mV})$ for every degree Celsius rise in the value of temperature. The resulting output potential can be transformed into temperature by means of the below expression. 


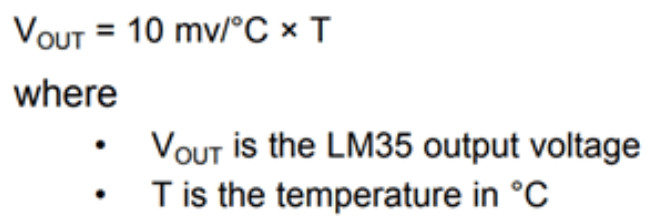

\section{iii) IC 741 OP AMP}

A monolithic silicon microchip, comprising a general purpose Operational Amplifier, is generally referred to as the $741 \mathrm{Op} \mathrm{Amp} \mathrm{IC.} \mathrm{The} \mathrm{first} \mathrm{manufacturers} \mathrm{of} \mathrm{the} 741 \mathrm{Op}$ Amp IC were Fairchild semiconductors in the year 1963. The name 741 specifies that this operational amplifier IC has 7 functional pins, out of which 4 pins are used to take input and 1 pin is used as output terminal.

IC 741 Op Amp is capable of producing high voltage amplification and therefore it can function over a extensive range of voltages. This property makes the IC 741 Op Amp, the best option for use in various applications such as integrators, summing amplifiers and general feedback applications. It also has some inbuilt distinct features like protective cover for short circuit and compensation circuits for internal frequency.

\section{iv) SURFACE ELECTRODES}

Surface electrodes are the electrodes that determine the bioelectric potential generated from the human body by placing them on the superficial layer of the skin. It observes the signal from heart, brain and nerves. Surface electrodes of larger size are used for sensing the ECG signals. Smaller surface electrodes are used for sensing the EMG and EEG signals. Certain category of the surface electrodes is capable of sensing the conductance of the body parts such as skin conductance or transthoracic impedance. Many varieties of electrodes are in existence. The type of surface electrode employed purely depends on the requirement of the appropriate application.

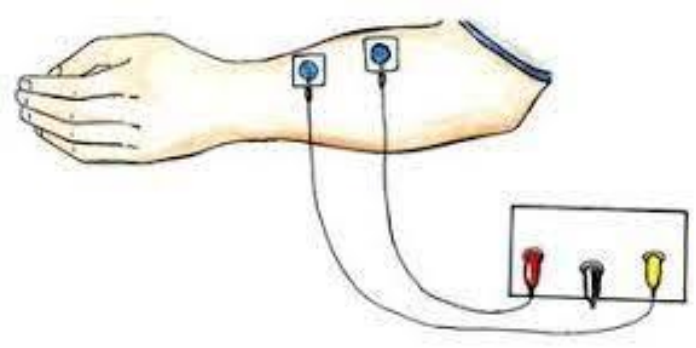

Fig 5. Surface Electrodes

\section{RESULTS AND DISCUSSION}

The figures 6 represents the measurement process of the bio signals from the subject. Figure 7 indicates Simulation output obtained from the limb electrodes. Figures 8 and 9 represent the consolidation of the simulations obtained from the proposed methodology. 


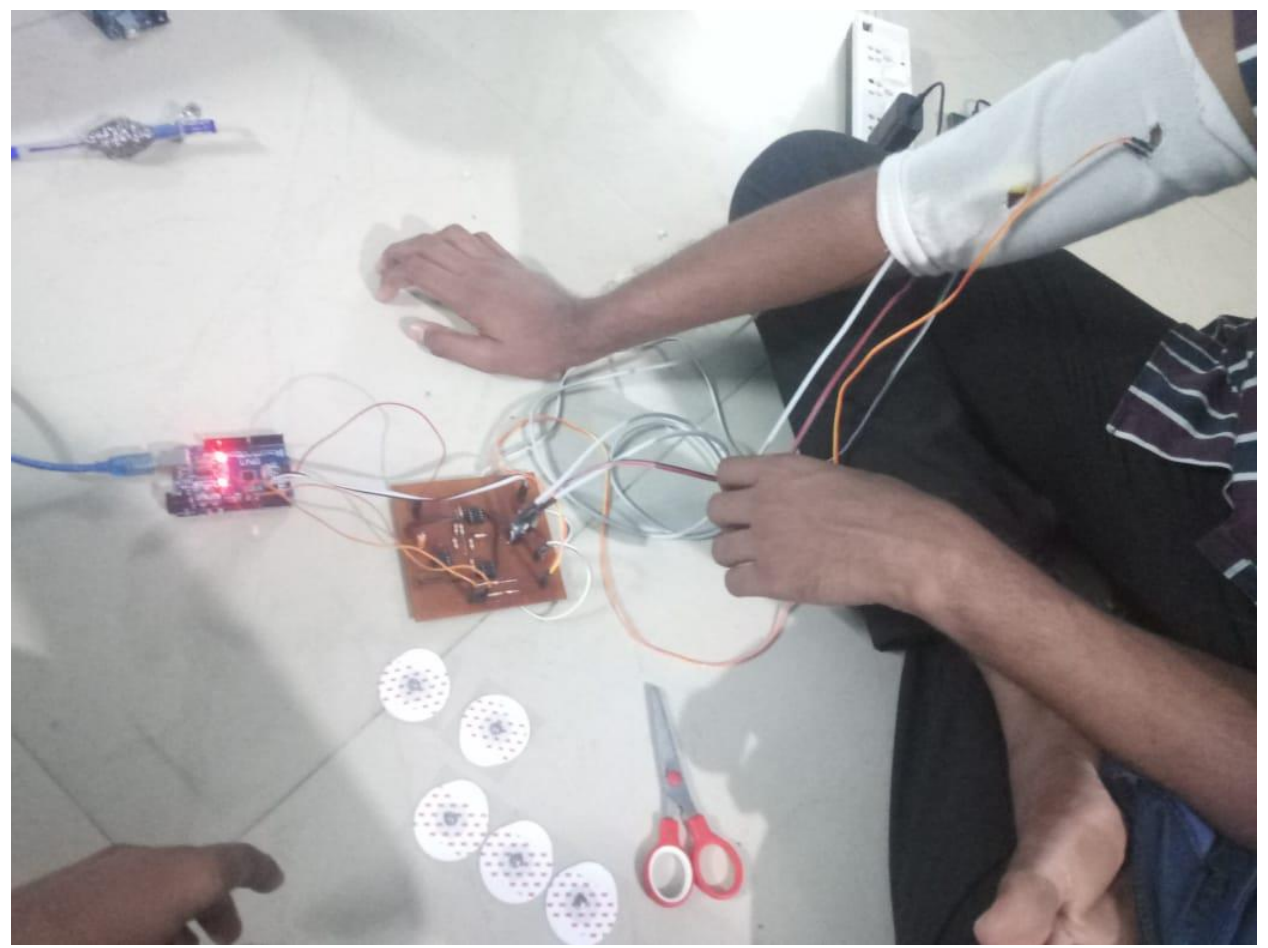

Fig 6. Measurement of biosignals using limp electrodes

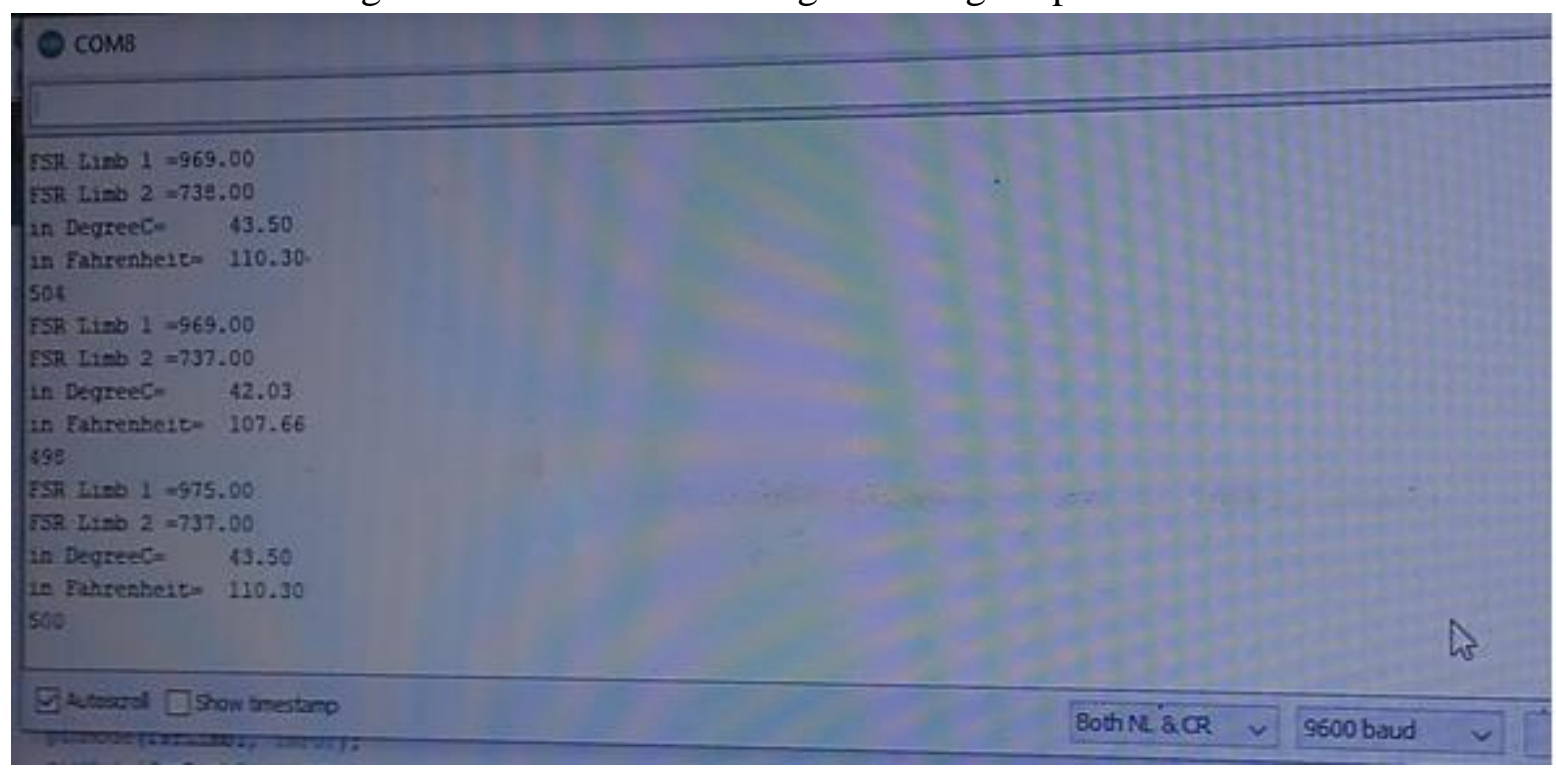

Fig 7. Simulation output from the limb electrodes

EMG SIGNAL OUTPUT - IN AMPLITUDE mv

TEMPERATURE - IN CELCIUS AND FARENHEIT

FLEX SENSOR- MOVEMENT BY POTENTIAL DIFFERENCE 


\begin{tabular}{|c|c|c|c|c|}
\hline FSR Limb $1=982.00$ & FSR Limb $2=746.00$ & Degree $C=34.21$ & Fahrenheit $=93.58$ & 582 \\
\hline FSR Limb $1=983.00$ & FSR Limb $2=745.00$ & Degree $C=36.17$ & Fahrenheit $=97.10$ & 626 \\
\hline FSR Limb $1=981.00$ & FSR Limb $2=745.00$ & Degree $C=32.26$ & Fahrenheit $=90.06$ & 541 \\
\hline FSR Limb $1=982.00$ & FSR Limb $2=745.00$ & Degree $C=39.10$ & Fahrenheit $=102.38$ & 632 \\
\hline FSR Limb $1=981.00$ & FSR Limb $2=745.00$ & Degree $C=32.75$ & Fahrenheit $=90.94$ & 575 \\
\hline FSR Limb $1=981.00$ & FSR Limb $2=745.00$ & Degree $C=34.21$ & Fahrenheit $=93.58$ & 586 \\
\hline FSR Limb $1=982.00$ & FSR Limb $2=745.00$ & Degree $C=36.66$ & Fahrenheit $=97.98$ & 633 \\
\hline FSR Limb $1=982.00$ & FSR Limb $2=745.00$ & Degree $C=31.77$ & Fahrenheit $=89.18$ & 548 \\
\hline FSR Limb $1=981.00$ & FSR Limb $2=745.00$ & Degree $C=40.57$ & Fahrenheit $=105.02$ & 638 \\
\hline FSR Limb $1=982.00$ & FSR Limb $2=745.00$ & Degree $C=32.26$ & Fahrenheit $=90.06$ & 577 \\
\hline FSR Limb $1=983.00$ & FSR Limb $2=745.00$ & Degree $C=35.68$ & Fahrenheit $=96.22$ & 586 \\
\hline FSR Limb $1=983.00$ & FSR Limb $2=746.00$ & Degree $C=36.17$ & Fahrenheit $=97.10$ & 638 \\
\hline FSR Limb $1=981.00$ & FSR Limb $2=744.00$ & Degree $C=30.30$ & Fahrenheit $=86.55$ & 545 \\
\hline FSR Limb $1=983.00$ & FSR Limb $2=745.00$ & Deqree $C=39.10$ & Fahrenheit $=102.38$ & 633 \\
\hline
\end{tabular}

Fig. 8. Measurement obtained at regular intervals

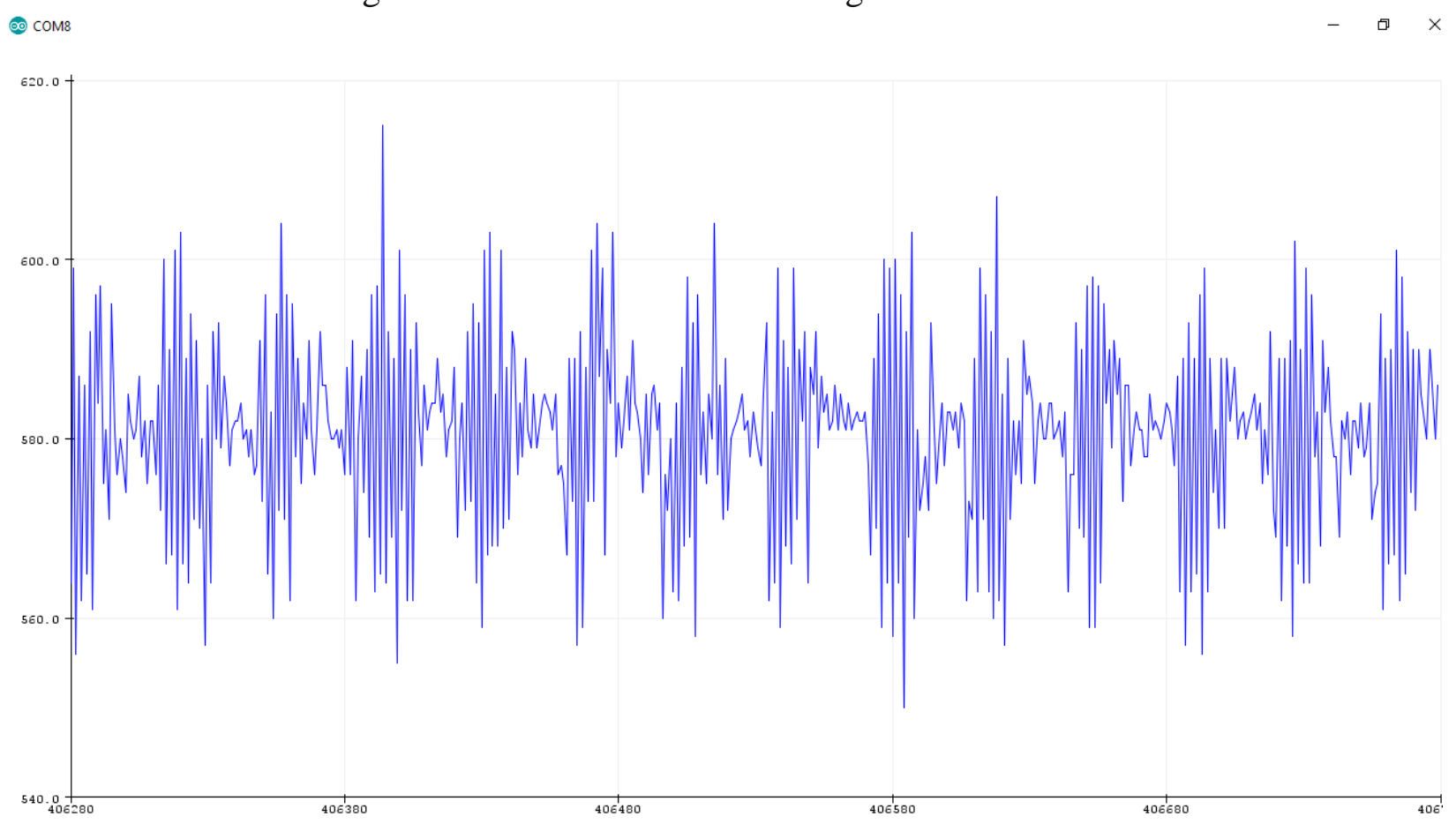

Fig.9. Consolidation of the biosignals obtained from the proposed system

\section{CONCLUSION}

The risk factors in a sports person such as high intensity work outs, muscle fatigue and prolonged running may cause muscle cramps or spasms. The electronic components which are mentioned in the proposed system are insulated and fixed in a vest (unisex). The vest is 
fabricated such that the damage of components and uncomfortable conditions occurring possibilities are very much reduced. Therefore with the help of this wearable device, muscle cramps occurring at upper limbs can be detected and further injuries, complications can be reduced. The proposed muscular vest also initiates early detection of muscle cramps, thereby avoiding the risk of fractures and injuries.

\section{REFERENCES}

1. Muscle Cramps, 2005, TIMOTHY M. MILLER, MD, PhD,1 and ROBERT B. LAYZER, MD2 1 Department of Neurosciences, University of California, San Diego, 9500 Gilman Drive, La Jolla, California 92093-0670, USA 2 Department of Neurology, University of California, San Francisco, California, USA

2. Origin and development of muscle cramps, 2013, MA Minetto, A Holobar, $\underline{\text { A Botter- }}$ Exercise and sports 2013

3. Exercise-Associated Muscle Cramps, 2010, Kevin C. Miller, PhD, ATC, CSCS, ${ }^{* \dagger}$ Marcus S. Stone, PhD, ATC, ${ }^{\star}$ Kellie C. Huxel, PhD, ATC, ${ }^{\S}$ and Jeffrey E. Edwards,Sports health, vol.2(4), pg. 279-283

4.Exercise-induced muscle pain, soreness, and cramps. 1994, MP Miles, PM Clarkson - The Journal of sports medicine, vol. 34(3), pg.203-216

5. Muscle Cramps, 2019, Michael C. Levin, College of Medicine, University of Saskatchewan 6.Muscle cramps: A comparison of the two-leading hypothesis, 2018, GaiaGiuriato ${ }^{a}$ AnnaPedrinolla ${ }^{a}$ FedericoSchena ${ }^{a}$ MassimoVenturelliab ${ }^{a}$, Journal of Electromyography and Kinesiology, Vol.41,Pg 89-95

7.Cramps, muscle pains and fasciculations, Not always benigh?, 2004, Mamede de Carvalho, Michael Swash, Neurology, vol. 63(4)

8. A randomized trial of mexiletine in ALS, 2016, Michael D. Weiss, Eric A. Macklin, Zachary Simmons, Angela S. Knox, David J. Greenblatt, NazemAtassi, Michael Graves, Nicholas Parziale, Johnny S. Salameh, Colin Quinn, Robert H. Brown, Jane B. Distad, Jaya Trivedi, Jeremy M. Shefner, Richard J. Barohn, Alan Pestronk, Andrea Swenson, Merit E. Cudkowicz, For the Mexiletine ALS Study Group, Neurology, vol. 86(16)

9.Tirapazamine Plus Cisplatin Versus Cisplatin in Advanced Non-Small-Cell Lung Cancer: A Report of the International CATAPULT I Study Group, 2000, Joachim von Pawel, Reinhard von Roemeling, Ulrich Gatzemeier, Michael Boyer, Lars OveElisson, Peter ClarkDenis Talbot, Augustin Rey, Thomas W. Butler, Vera Hirsh, Ian Olver, Bengt Bergman, Joseph Ayoub, Gary Richardson, David Dunlop, Anthony Arcenas, Robert Vescio, Jean Viallet, Joseph Treat, Journal of Clinical Oncology, vol. $18(6)$

10. Riikonen, Raili, The latest on infantile spasms,2005, , Current opinion in Neurology, vol. 18(2), pg. 91-95

11. Miller, Kevin C. Ph.D., AT, The Evolution of Exercise-Associated Muscle Cramp Research, ATC

ACSM's Health \& Fitness Journal: July/August 2018 - Volume 22 - Issue 4 - p 6-8

12. Sontag SJ, Wanner JN, The cause of leg cramps and knee pains: an hypothesis and effective treatment, Med Hypotheses 1988;25:35-41

13. Helin P, Physiotherapy and electromyography in muscle cramp. , Br J Sports Med 1985; 19:230-1. 
14. Nelson LD, Hutton RS, Dynamic and static stretch response in muscle spindle receptors in fatigued muscle, Med Sci Sports Exerc 1985;17:445-50.

15. Hutton RS, Nelson LD, Stretch sensitivity of Golgi tendon organs in fatigued gastrocnemius muscle,

Med Sci Sports Exerc 1986; 18:69-74.

16. Baldissera F, Cavallari P, Dworzak F, Motor neuron 'bistability'. A pathogenetic mechanism for cramps and myokymia, Brain 1994; 117(Pt 5):929-39.

17. Minetto MA, Botter A, Ravenni R, et al, Reliability of a novel neurostimulation method to study involuntary muscle phenomena, ,Muscle Nerve 2008;37:90-100.

18. han SI, Burne JA, Reflex inhibition of normal cramp following electrical stimulation of the muscle tendon, , J Neurophysiol 2007;98:1102-7.

19. Ross BH,Thomas CK,. Human motor unit activity during induced muscle cramp. Brain 1995;118:983-93.

20. Manjra SI, Schwellnus MP, Noakes TD, Risk factors for exercise associated muscle cramping (EAMC) in marathon runners. Med Sci Sports Exerc 1996;28 